Dhaka Univ. J. Biol. Sci. 30(1): 79-86, 2021 (January)

\title{
EFFECTS OF ORGANIC MANURES ON THE GROWTH AND YIELD OF MALABAR NUT (JUSTICIA ADHATODA L.)
}

\author{
S RAHMAN*, KN NiSHI, M MUSLIM AND MK RAHMAN \\ Department of Soil, Water and Environment, University of Dhaka, \\ Dhaka-1000, Bangladesh
}

Key words: Growth and yield, Organic manures, Malabar nut

\begin{abstract}
A pot $(7 \mathrm{~kg}$ soil/pot) experiment was carried out to evaluate the growth performance of malabar nut (Justicia adhatoda L.) as influenced by different organic manures. Manures were (a) ACI, (b) FDA, (c) GTS, (d) Green life, (e) Kaji, (f) Majim, (g) Shebok, (h) Shufola, (i) Cowdung, and (j) Water hyacinth, applied at the rate of 10 ton/ha. There were eleven treatments and three replications. Pots were arranged in a completely randomized design (CRD). To evaluate the growth performance viz. height, leaf number, leaf area, and leaf area index, girth, and branches were recorded at $15 \mathrm{~d}$ intervals up to harvest. Plants ( $90 \mathrm{~d}$ old) were harvested as root, stem, and leaf. Fresh and dry weights of root, stem, and leaf were recorded. The highest height $(80.90 \mathrm{~cm})$, leaf number (28.70 no./plant), leaf area $\left(175.77 \mathrm{~cm}^{2}\right.$ plant), leaf area index $(0.462)$ were observed in water hyacinth. The lowest height $(42.20 \mathrm{~cm})$, leaf number (13.70 no.plant), leaf area $\left(45.58 \mathrm{~cm}^{2}\right.$ /plant), and leaf area index (0.12) were recorded in control. Maximum fresh and dry weights (root, stem, and leaf) were $129.19 \mathrm{~g} / \mathrm{plant}$ and $57.97 \mathrm{~g} / \mathrm{plant}$ found in water hyacinth, respectively. Results concluded that the overall growth and yield were achieved better in water hyacinth manure.
\end{abstract}

\section{Introduction}

Malabar nut (Justicia adhatoda L.) known as Vasaka plant in Bengali, belongs to the family Acanthaceae. The leaves, roots, and young plants of Vasaka plant contain the quinazoline alkaloids vasicine, 7-hydroxy vasicine, vasicinolone, 3-deoxy vasicine, vasicol, vasicoline,vasicinone, triterpenes, anisotine, betaine, steroids, carbohydrate and alkanes ${ }^{(1,2)}$. It is a perennial shrub small, evergreen plant which reaches an average height of three meters. Its branches are opposite and ascending. The broad leaves, which are sometimes used as an insecticide, measure from 10 to $15 \mathrm{~cm}$ in length, and are about $4 \mathrm{~cm}$ in width. Malabar nut grows in all parts of the worlds and the bark, flowers, roots, and leaves are used in medicine. Malabar nut, native to Asia is a well-known plant for Siddha, Ayurveda, and Unani systems of medicine. Various parts of this plant have been used to treat several ailments as herbal remedies such as cold, cough, whooping cough, chronic bronchitis, fever, jaundice, asthma as a sedative expectorant, diarrhea and

*Author for correspondence: <sharminrahmanantora@gmail.com>. DOI: https://doi.org/10.3329/dujbs.v30i1.51811 
dysentery and rheumatic painful inflammatory swellings $\mathrm{s}^{(3,4,5)}$. Malabar nut is used to control both internal and external bleeding such as aspiles, peptic ulcers, and bleeding gums. It exhibits expectorant, antispasmodic and blood purifying qualities. The extract of roots of Malabar nut is commonly used by the rural population against diabetes, cough, and certain liver disorders ${ }^{(6)}$. The leaves are rich in vitamin $C$ and carotene and yield in essential oils. Chemical compounds found in leaves and roots of this plant include essential oils, fats, resins, sugar, gum, amino acids, proteins, and vitamin C, etc. ${ }^{(7)}$. The phytochemical studies of the various parts of Malabar nut revealed the presence of polyphenolics, alkaloids, phytosterols, and glycosides as a major class of compound.

The whole plant is used as an ingredient of numerous popular formulations including cough syrup used in combination with ginger (Zingiber officinale L.) and tulsi (Ocimum sanctum L.) where it exerts its action as an expectorant and antispasmodic ${ }^{(8)}$. The various preparation of leaves is used for curing bleeding, haemorrhage, skin diseases, wounds, headache, and leprosy in Southeast Asia(9). The paste, powder, and digestion of root are used for curing tuberculosis, diphtheria, malarial fever, leucorrhoea, and eye diseases in Southeast Asia(10). Malabar nut was studied for its anti-ulcerogenic activity against ulcers induced by ethanol, pylorus, and aspirin. It can also be found in rural areas along with roadside, riverside and the tree is used as a fence for villagers' vegetable fields. The monsoon period is favorable for the growth of Malabar nut. The plant is generally cultivated in sandy soil. The use of organic matter in combination with inorganic fertilizers to optimize nutrient availability to plants is a difficult task as organic materials have complex chemical nature. This requires the understanding and knowledge of the chemical composition, particularly the nutrient content and quality of organic materials and its interaction with inorganic nutrient sources. The use of organic manure could be an inevitable practice in the coming years for ensuring suitable crop productivity without affecting soil fertility ${ }^{(11,12)}$. A number of companies in Bangladesh are producing organic manures using natural resources and farmers are using them without any potfield trial. Malabar nut is generally found in the southern part of Bangladesh. Recently, a number of pharmaceutical companies of Bangladesh are using Malabar nut as a raw material for medicinal purposes. Scanty information is available in the literature for the cultivation of Malabar nut plants through either organic or inorganic farming under Bangladesh edaphic conditions.

Thus, the objective of the experiment was to evaluate the growth and yield of Malabar nut as influenced by different organic manures.

\section{Material and Methods}

Soil sample (0 to $15 \mathrm{~cm}$ depth) was collected from Dendabor, Savar upazila under Dhaka division. The sample was air-dried, ground, and sieved through $2 \mathrm{~mm}$ sieve. The soil had a $\mathrm{pH}$ of 6.72 , organic carbon $0.56 \%{ }^{(13)}$, available N $21 \mathrm{mg} / \mathrm{kg}^{(14)}$, available P 5.8 
$\mathrm{mg} / \mathrm{kg}^{(15)}$, available $\mathrm{K} 31.1 \mathrm{mg} / \mathrm{kg}$, field capacity $25 \%{ }^{(16)}$ sand $8.12 \%$, silt $52.34 \%$ and clay $39.54 \%$, and textural class-silt loam. Seven kilograms of soil were taken per plastic pot (30 $\mathrm{cm} \times 20 \mathrm{~cm}$ ). Manures from different companies like ACI, FDA, GTS, Green Life, Kaji, Majim, Shebok, Shufola, Cowdung, and Water hyacinth were bought from the local market and applied at the rate of 10 ton/ha. Pots were arranged in a completely randomized design (CRD). Four weeks old a seedling of Malabar nut was transplanted per pot. There were eleven treatments and three replications. The pots were watered in the morning. Height was measured from the soil level to the tip of the leaf and leaf number was counted with the help of a tally counter at 15day intervals. Leaf area index was determined according to the following formula:

$$
\text { Leaf area index: } \frac{\text { leaf area }}{\text { Ground area }}
$$

Three months old plants were harvested as root, stem and leaf, washed with tap water and finally with distilled water, and wrapped with soft tissue paper. Immediately after harvest fresh weights were taken and then air-dried in the room temperature for 48 hours. Dry weight $\left(65^{\circ} \mathrm{C}\right)$ of the samples was recorded and grounded in a mechanical grinder and stored in paper bags for chemical analysis. Results were statistically analyzed using Microsoft Excel 2013 and Software Minitab 19.

\section{Results and Discussion}

Plant growth: Growth was evaluated in terms of plant height (Table 1), leaf number (Table 2), leaf area and leaf area index (Table 3), girth and branch (Table 4), and fresh and dry weights of root, stem and leaf (Table 5). Differences were determined by one-way ANOVA followed by Tukey's pairwise comparison $(\mathrm{p}<0.05)$. Different letters in the same row indicate significant differences between treatments.

Height: At harvest, plant height were 42.20, 43.70, 49.50, 44.40, 66.30, 58.90, 63.80, 58.60, 65.05, 66.60 and $80.90 \mathrm{~cm}$ in control, ACI, FDA, GTS, Green life, Kaji, Majim, Shebok, Shufola, Cowdung and Water hyacinth treatments, respectively (Table 1). The highest length of plant was $80.90 \mathrm{~cm}$ observed in water hyacinth treatment and the lowest value $42.20 \mathrm{~cm}$ was found in control treatment. Water hyacinth contains a number of macro- and micro nutrients, and maintains good physical properties of the soil that probably played the significant role for the better growth of Malabar nut. Height varied significantly $(\mathrm{p}<0.05)$ at harvest over control (Table 1$)$.

Leaf number: Number of leaf per plant responded positively following all treatments. After 90 days the leaf number found from the eleven treatments were 9.10, 16.50, 12.30, $15.70,24.30,18.70,21.00,27.30,21.00,22.70$ and 28.70 in eleven treatments, respectively (Table 2). The highest number of leaf were achieved 28.7 in water hyacinth and the 
lowest value 9.10 was found in control treatment (Table 2). The organic manures contain a passive fraction of 60 to 90 per cent which is very slowly available to plants. Therefore, it might be possible that organic manures doses of the treatment contained comparatively larger portion of passive fraction and it took a long time to release nutrients. The number of leaf per plant at harvest varied significantly $(5 \varangle 0.05)$ (except FDA compost) over control (Table 2)

Table 1. Effects of different organic manures on height of Malabar nut at 15 days interval.

\begin{tabular}{lccccc}
\hline \multirow{5}{*}{ Treatments } & $30 \mathrm{~d}$ & $45 \mathrm{~d}$ & $60 \mathrm{~d}$ & $75 \mathrm{~d}$ & $90 \mathrm{~d}$ \\
\cline { 2 - 6 } Control(-OM) & $31.30^{\mathrm{h}}$ & $33.0^{\mathrm{h}}$ & $33.27^{\mathrm{bc}}$ & $34.20^{\mathrm{k}}$ & $42.20^{\mathrm{i}}$ \\
ACI & $32.50^{\mathrm{f}}$ & $34.00^{\mathrm{g}}$ & $36.80^{\mathrm{ab}}$ & $38.0^{\mathrm{j}}$ & $43.70^{\mathrm{h}}$ \\
FDA & $38.50^{\mathrm{d}}$ & $43.00^{\mathrm{d}}$ & $42.70^{\mathrm{ab}}$ & $45.60^{\mathrm{h}}$ & $49.50^{\mathrm{f}}$ \\
GTS & $39.00^{\mathrm{d}}$ & $43.30^{\mathrm{d}}$ & $44.03^{\mathrm{ab}}$ & $43.20^{\mathrm{i}}$ & $44.40^{\mathrm{g}}$ \\
Green life & $36.80^{\mathrm{e}}$ & $39.70^{\mathrm{f}}$ & $39.03^{\mathrm{ab}}$ & $54.70^{\mathrm{e}}$ & $66.30^{\mathrm{b}}$ \\
Kaji & $39.80^{\mathrm{c}}$ & $33.90^{\mathrm{i}}$ & $40.40^{\mathrm{ab}}$ & $48.96^{\mathrm{g}}$ & $58.90^{\mathrm{e}}$ \\
Majim & $39.50^{\mathrm{d}}$ & $47.00^{\mathrm{c}}$ & $50.70^{\mathrm{ab}}$ & $62.90^{\mathrm{b}}$ & $63.80^{\mathrm{d}}$ \\
Shebok & $41.90^{\mathrm{b}}$ & $48.40^{\mathrm{a}}$ & $50.90^{\mathrm{ab}}$ & $60.20^{\mathrm{c}}$ & $58.60^{\mathrm{e}}$ \\
Shufola & $34.70^{\mathrm{g}}$ & $41.50^{\mathrm{e}}$ & $41.75^{\mathrm{ab}}$ & $57.45^{\mathrm{d}}$ & $65.05^{\mathrm{c}}$ \\
Cowdung & $47.80^{\mathrm{a}}$ & $47.70^{\mathrm{b}}$ & $49.03^{\mathrm{ab}}$ & $54.10^{\mathrm{f}}$ & $66.60^{\mathrm{b}}$ \\
Water hyacinth & $24.50^{\mathrm{i}}$ & $48.90^{\mathrm{a}}$ & $52.80^{\mathrm{a}}$ & $70.80^{\mathrm{a}}$ & $80.90^{\mathrm{a}}$ \\
\hline
\end{tabular}

abcdefghi Data bearing different superscripts within the same column differ significantly at 5\% level.

Leaf area and leaf area index: At harvest, the leaf area were 60.58, 64.38, 64.50, 71.66, 148.20, 129.72, 128.47, 169.06, 166.16, 104.32 and, $175.77 \mathrm{~cm}^{2}$ plant in Control, ACI, FDA, GTS, Green life, Kaji, Majim, Shebok, Shufola, Cowdung and Water hyacinth, respectively (Table 3). Highest leaf area $175.77 \mathrm{~cm}^{2}$ was found in water hyacinth and the lowest value $60.58 \mathrm{~cm}^{2}$ was recorded in control treatment. After 90 days the leaf index found from the eleven treatments were $0.120,0.169,0.170,0.189,0.390,0.341,0.338,0.445$, $0.437,0.274$, and 0.462 , respectively (Table 3). Highest leaf area index 0.462 was found in water hyacinth and the lowest value was obtained 0.120 in control treatment. Leaf area and leaf area index varied significantly $(\mathrm{p}<0.5)$ at harvest over control (Table 3).

Girth and branch: At harvest, the girth recorded from the eleven treatments were 2.20, $3.58,3.97,3.90,3.00,3.40,3.87,3.53,4.00,4.60$ and $4.30 \mathrm{~cm}$, respectively (Table 4). The highest value $4.60 \mathrm{~cm}$ was found in cow dung and the lowest value $2.20 \mathrm{~cm}$ was recorded in control treatment. Cow dung compost can be added directly to the soil without damaging plants. It also adds plentiful amounts of organic matter and beneficial 
microorganisms. After 90 days the branches found from the eleven treatments were 1.60, $2.40,2.41,2.40,2.10,2.10,2.30,2.20,2.17,2.40$, and 2.44 no./plant. The highest number of branches 2.44 was found in water hyacinth and the lowest value 1.60 was observed in control (Table 4). The values of girth and number of branches per plant were varied significantly $(\mathrm{p}<0.05)$ at harvest over control (Table 4$)$.

Table 2. Effects of different organic manures on leaf number of Malabar nut at 15 days interval.

\begin{tabular}{lcccccc}
\hline \multirow{2}{*}{ Treatments } & \multicolumn{5}{c}{ Leaf number } \\
\cline { 2 - 6 } & $15 \mathrm{~d}$ & $30 \mathrm{~d}$ & $45 \mathrm{~d}$ & $60 \mathrm{~d}$ & $75 \mathrm{~d}$ & $90 \mathrm{~d}$ \\
\hline Control (-OM) & $4.0^{\mathrm{f}}$ & $5.12^{\mathrm{f}}$ & $6.50^{\mathrm{d}}$ & $7.10^{\mathrm{g}}$ & $8.40^{\mathrm{ef}}$ & $9.10^{\mathrm{h}}$ \\
ACI & $9.00^{\mathrm{e}}$ & $11.70^{\mathrm{cd}}$ & $9.50^{\mathrm{cd}}$ & $8.30^{\mathrm{fg}}$ & $13.50^{\mathrm{cd}}$ & $16.50^{\mathrm{ef}}$ \\
FDA & $6.70^{\mathrm{f}}$ & $7.70^{\mathrm{e}}$ & $9.30^{\mathrm{cd}}$ & $11.50^{\mathrm{de}}$ & $9.50^{\mathrm{e}}$ & $12.30 \mathrm{~g}^{\mathrm{h}}$ \\
GTS & $4.70^{\mathrm{h}}$ & $12.30^{\mathrm{bc}}$ & $8.70^{\mathrm{de}}$ & $9.70^{\mathrm{ef}}$ & $16.00^{\mathrm{bc}}$ & $15.70^{\mathrm{fg}}$ \\
Green life & $13.30^{\mathrm{a}}$ & $16.00^{\mathrm{ab}}$ & $15.70^{\mathrm{a}}$ & $14.30^{\mathrm{bc}}$ & $18.30^{\mathrm{ab}}$ & $24.30^{\mathrm{b}}$ \\
Kaji & $9.30^{\mathrm{e}}$ & $11.70^{\mathrm{cd}}$ & $9.30^{\mathrm{cd}}$ & $10.70^{\mathrm{def}}$ & $13.00^{\mathrm{cd}}$ & $18.70^{\mathrm{de}}$ \\
Majim & $6.70^{\mathrm{f}}$ & $9.00^{\mathrm{cde}}$ & $11.00^{\mathrm{c}}$ & $12.70^{\mathrm{cd}}$ & $18.30^{\mathrm{ab}}$ & $21.00^{\mathrm{cd}}$ \\
Shebok & $13.70^{\mathrm{a}}$ & $18.00^{\mathrm{a}}$ & $17.30^{\mathrm{a}}$ & $19.00^{\mathrm{a}}$ & $21.70^{\mathrm{a}}$ & $27.30^{\mathrm{a}}$ \\
Shufola & $6.00^{\mathrm{g}}$ & $8.00^{\mathrm{de}}$ & $7.50^{\mathrm{e}}$ & $8.00^{\mathrm{g}}$ & $10.00^{\mathrm{de}}$ & $21.00^{\mathrm{cd}}$ \\
Cowdung & $11.70^{\mathrm{c}}$ & $17.70^{\mathrm{a}}$ & $16.60^{\mathrm{a}}$ & $16.70^{\mathrm{ab}}$ & $17.70^{\mathrm{b}}$ & $22.70^{\mathrm{bc}}$ \\
Water & $10.30^{\mathrm{d}}$ & $18.70^{\mathrm{a}}$ & $17.30^{\mathrm{a}}$ & $16.30^{\mathrm{b}}$ & $13.30^{\mathrm{cd}}$ & $28.70^{\mathrm{a}}$ \\
hyacinth & & & & & \\
\hline
\end{tabular}

a bcdefg Data bearing different superscripts within the same column differ significantly at 5\% level.

Yield parameters: The highest fresh weight of root per plant was $40.7 \mathrm{~g}$ found in cow dung and lowest was $5.40 \mathrm{~g}$ found in control. The highest fresh weight of stem per plant was $60.05 \mathrm{~g}$ found in water hyacinth and lowest was $11.13 \mathrm{~g}$ found in control. The highest fresh weight of leaf per plant was $43.02 \mathrm{~g}$ observed in water hyacinth and that of the lowest was $3.25 \mathrm{~g}$ found in control. After 90 days the total fresh weight found from the eleven treatments were 19.80, 39.79, 24.67, 32.79, 68.78, 80.20, 120.87, 105.34, 52.14, 126.08, and $129.19 \mathrm{~g}$, respectively. The highest total fresh weight of a plant was $129.19 \mathrm{~g}$ recorded in water hyacinth and lowest was $19.80 \mathrm{~g}$ found in control treatment (Table 5). Results of total fresh and dry weight of a plant varied significantly $(p<0.05)$ at harvest over the control (Table 5). Water hyacinth has drawn attention as a plant of rapid growth and high biomass production, and capable of removing pollutants from domestic and industrial waste effluents ${ }^{(17)}$.

The highest dry weight of root per plant was $16.04 \mathrm{~g}$ recorded in water hyacinth and lowest was $1.20 \mathrm{~g}$ found in control. The highest dry weight of stem per plant was $27.68 \mathrm{~g}$ 
Table 3. Effects of different organic manures on leaf area $\left(\mathrm{cm}^{2} / \mathrm{plant}\right)$ and leaf area index of Malabar nut at 15 days interval.

\begin{tabular}{lcccccccc}
\hline \multirow{2}{*}{ Treatments } & \multicolumn{3}{c}{ Leaf area $\left(\mathrm{cm}^{2}\right)$} & \multicolumn{4}{c}{ Leaf area index } \\
\cline { 2 - 9 } & $45 \mathrm{~d}$ & $60 \mathrm{~d}$ & $75 \mathrm{~d}$ & $90 \mathrm{~d}$ & $45 \mathrm{~d}$ & $60 \mathrm{~d}$ & $75 \mathrm{~d}$ & $90 \mathrm{~d}$ \\
\hline Control (-OM) & $27.17^{\mathrm{j}}$ & $29.78^{\mathrm{k}}$ & $29.54^{\mathrm{k}}$ & $60.58^{\mathrm{j}}$ & $0.105^{\mathrm{i}}$ & $0.112^{\mathrm{j}}$ & $0.118^{\mathrm{k}}$ & $0.120^{\mathrm{j}}$ \\
ACI & $39.83^{\mathrm{i}}$ & $36.73^{\mathrm{j}}$ & $42.96^{\mathrm{j}}$ & $64.38^{\mathrm{i}}$ & $0.124^{\mathrm{h}}$ & $0.130^{\mathrm{k}}$ & $0.156^{\mathrm{j}}$ & $0.169^{\mathrm{i}}$ \\
FDA & $38.25^{\mathrm{i}}$ & $42.50^{\mathrm{j}}$ & $46.62^{\mathrm{h}}$ & $64.50^{\mathrm{i}}$ & $0.123^{\mathrm{h}}$ & $0.132^{\mathrm{i}}$ & $0.158^{\mathrm{j}}$ & $0.170^{\mathrm{i}}$ \\
GTS & $50.14^{\mathrm{g}}$ & $46.04^{\mathrm{i}}$ & $91.68^{\mathrm{f}}$ & $71.66^{\mathrm{h}}$ & $0.132^{\mathrm{g}}$ & $0.141^{\mathrm{i}}$ & $0.240^{\mathrm{i}}$ & $0.189^{\mathrm{h}}$ \\
Green life & $59.80^{\mathrm{e}}$ & $58.93^{\mathrm{f}}$ & $100.44^{\mathrm{e}}$ & $148.20^{\mathrm{d}}$ & $0.157^{\mathrm{e}}$ & $0.158^{\mathrm{f}}$ & $0.264^{\mathrm{e}}$ & $0.390^{\mathrm{d}}$ \\
Kaji & $54.38^{\mathrm{f}}$ & $60.14^{\mathrm{e}}$ & $82.15^{\mathrm{g}}$ & $129.72^{\mathrm{e}}$ & $0.143^{\mathrm{f}}$ & $0.158^{\mathrm{e}}$ & $0.261^{\mathrm{g}}$ & $0.341^{\mathrm{e}}$ \\
Majim & $86.73^{\mathrm{c}}$ & $102.06^{\mathrm{b}}$ & $179.068^{\mathrm{a}}$ & $128.47^{\mathrm{f}}$ & $0.228^{\mathrm{c}}$ & $0.268^{\mathrm{b}}$ & $0.471 \mathrm{a}$ & $0.338^{\mathrm{f}}$ \\
Shebok & $89.09^{\mathrm{b}}$ & $82.88^{\mathrm{c}}$ & $119.83^{\mathrm{d}}$ & $169.06^{\mathrm{b}}$ & $0.234^{\mathrm{b}}$ & $0.238^{\mathrm{c}}$ & $0.315^{\mathrm{d}}$ & $0.445^{\mathrm{b}}$ \\
Shufola & $51.08^{\mathrm{g}}$ & $47.08^{\mathrm{h}}$ & $122.27^{\mathrm{c}}$ & $166.16^{\mathrm{c}}$ & $0.134^{\mathrm{g}}$ & $0.124^{\mathrm{h}}$ & $0.322^{\mathrm{c}}$ & $0.437^{\mathrm{c}}$ \\
Cowdung & $78.00^{\mathrm{d}}$ & $72.36^{\mathrm{d}}$ & $81.081^{\mathrm{h}}$ & $104.32^{\mathrm{g}}$ & $0.205^{\mathrm{d}}$ & $0.190^{\mathrm{d}}$ & $0.213^{\mathrm{h}}$ & $0.274^{\mathrm{g}}$ \\
Water hyacinth & $107.25^{\mathrm{a}}$ & $121.98^{\mathrm{a}}$ & $163.02^{\mathrm{b}}$ & $175.77^{\mathrm{a}}$ & $0.282^{\mathrm{a}}$ & $0.320^{\mathrm{c}}$ & $0.429^{\mathrm{b}}$ & $0.462^{\mathrm{a}}$ \\
\hline
\end{tabular}

a b cdefghijk Data bearing different superscripts within the same column differ significantly at 5\% level.

Table 4. Effects of organic manures on girth and branch of Malabar nut at 15 days interval.

\begin{tabular}{lllllllll}
\hline \multirow{2}{*}{ Treatments } & \multicolumn{4}{c}{ Girth $(\mathrm{cm})$} \\
\cline { 2 - 8 } & $45 \mathrm{~d}$ & $60 \mathrm{~d}$ & $75 \mathrm{~d}$ & $90 \mathrm{~d}$ & $45 \mathrm{~d}$ & $60 \mathrm{~d}$ & $75 \mathrm{~d}$ & $90 \mathrm{~d}$ \\
\hline Control(-OM) & $1.50^{\mathrm{e}}$ & $1.90^{\mathrm{f}}$ & $2.05^{\mathrm{e}}$ & $2.20^{\mathrm{h}}$ & $1.10^{\mathrm{d}}$ & $1.35^{\mathrm{bc}}$ & $1.50^{\mathrm{d}}$ & $1.60^{\mathrm{c}}$ \\
ACI & $3.25^{\mathrm{de}}$ & $3.37^{\mathrm{ef}}$ & $3.50^{\mathrm{de}}$ & $3.58 \mathrm{~g}$ & $2.33^{\mathrm{b}}$ & $2.34^{\mathrm{a}}$ & $2.38^{\mathrm{a}}$ & $2.40^{\mathrm{ab}}$ \\
FDA & $3.00^{\mathrm{de}}$ & $3.30^{\mathrm{cd}}$ & $3.50^{\mathrm{de}}$ & $3.97^{\mathrm{f}}$ & $2.34^{\mathrm{a}}$ & $2.34^{\mathrm{a}}$ & $2.38^{\mathrm{a}}$ & $2.41^{\mathrm{ab}}$ \\
GTS & $3.17^{\mathrm{de}}$ & $3.67^{\mathrm{e}}$ & $3.80^{\mathrm{cd}}$ & $3.90^{\mathrm{fg}}$ & $2.33^{\mathrm{b}}$ & $2.33^{\mathrm{a}}$ & $2.34^{\mathrm{ab}}$ & $2.40^{\mathrm{ab}}$ \\
Green life & $3.77^{\mathrm{a}}$ & $3.97^{\mathrm{de}}$ & $3.43^{\mathrm{b}}$ & $3.00^{\mathrm{ef}}$ & $2.00^{\mathrm{c}}$ & $2.04^{\mathrm{b}}$ & $2.10^{\mathrm{b}}$ & $2.10^{\mathrm{ab}}$ \\
Kaji & $3.13^{\mathrm{de}}$ & $3.17^{\mathrm{e}}$ & $3.27^{\mathrm{bc}}$ & $3.40^{\mathrm{de}}$ & $2.045^{\mathrm{b}}$ & $2.10^{\mathrm{ab}}$ & $2.10^{\mathrm{b}}$ & $2.10^{\mathrm{ab}}$ \\
Majim & $3.57^{\mathrm{bc}}$ & $3.73^{\mathrm{ab}}$ & $3.87^{\mathrm{ab}}$ & $3.87^{\mathrm{bc}}$ & $1.40^{\mathrm{c}}$ & $2.10^{\mathrm{ab}}$ & $2.14^{\mathrm{b}}$ & $2.30^{\mathrm{b}}$ \\
Shebok & $3.27^{\mathrm{de}}$ & $3.30^{\mathrm{cd}}$ & $3.37^{\mathrm{b}}$ & $3.53^{\mathrm{cd}}$ & $2.10^{\mathrm{b}}$ & $2.10^{\mathrm{b}}$ & $2.17^{\mathrm{c}}$ & $2.20^{\mathrm{ab}}$ \\
Shufola & $3.75^{\mathrm{a}}$ & $3.85^{\mathrm{a}}$ & $3.95^{\mathrm{a}}$ & $4.00^{\mathrm{bc}}$ & $2.05^{\mathrm{b}}$ & $2.10^{\mathrm{ab}}$ & $2.17^{\mathrm{c}}$ & $2.17^{\mathrm{ab}}$ \\
Cowdung & $3.07^{\mathrm{e}}$ & $3.13^{\mathrm{bc}}$ & $4.00^{\mathrm{a}}$ & $4.60^{\mathrm{a}}$ & $2.05^{\mathrm{b}}$ & $2.20^{\mathrm{ab}}$ & $2.34^{\mathrm{ab}}$ & $2.40^{\mathrm{ab}}$ \\
Water hyacinth & $2.63^{\mathrm{ef}}$ & $3.33^{\mathrm{cd}}$ & $3.97^{\mathrm{a}}$ & $4.30^{\mathrm{ab}}$ & $2.10^{\mathrm{b}}$ & $2.10^{\mathrm{ab}}$ & $2.27^{\mathrm{c}}$ & $2.44^{\mathrm{a}}$ \\
\hline
\end{tabular}

a bcdef Data bearing different superscripts within the same column differ significantly at $5 \%$ level.

observed in Water hyacinth and lowest was $2.40 \mathrm{~g}$ found in Control. The highest dry weight of leaf per plant was $15.4 \mathrm{~g}$ observed in Majim and that of the lowest was $1.84 \mathrm{~g}$ 
recorded in control. After 90 days the total dry weight achieved from the eleven treatments were 5.52, 13.21, 8.98, 11.19, 21.25, 30.73, 50.0, 31.95, 14.54, 38.82 and $57.97 \mathrm{~g}$, respectively (Table 5). The highest total dry weight of a plant was $57.97 \mathrm{~g}$ found in water hyacinth and lowest was $5.52 \mathrm{~g}$ observed in control treatment. The addition of water hyacinth manure into cultivation which affected the performance of test plant might be probably due to the increase of nitrogen availability released from water hyacinth during the process of mineralization ${ }^{(18)}$.

Table 5. Effect of organic manures on fresh weight and dry weight of root, stem and leaf of Malabar nut at 15 days interval.

\begin{tabular}{|c|c|c|c|c|c|c|c|c|}
\hline \multirow[b]{2}{*}{ Treatments } & \multicolumn{4}{|c|}{ Fresh weight (g/plant) } & \multicolumn{4}{|c|}{ Dry weight (g/plant) } \\
\hline & $\begin{array}{c}\text { Root } \\
\text { (g/plant) }\end{array}$ & $\begin{array}{c}\text { Stem } \\
\text { (g/plant) }\end{array}$ & $\begin{array}{c}\text { Leaf } \\
\text { (g/plant) }\end{array}$ & $\begin{array}{c}\text { Total } \\
\text { (g/plant) }\end{array}$ & $\begin{array}{c}\text { Root } \\
\text { (g/plant) }\end{array}$ & $\begin{array}{c}\text { Stem } \\
\text { (g/plant) }\end{array}$ & $\begin{array}{c}\text { Leaf } \\
\text { (g/plant) }\end{array}$ & $\begin{array}{c}\text { Total } \\
\text { (g/plant) }\end{array}$ \\
\hline Control(-OM) & $5.40^{\mathrm{i}}$ & $11.13^{j}$ & $3.25 j$ & $19.80^{\mathrm{k}}$ & $1.20^{\mathrm{i}}$ & $2.40^{j}$ & $1.84^{j}$ & $5.52^{\mathrm{h}}$ \\
\hline $\mathrm{ACI}$ & $6.00^{\mathrm{h}}$ & $23.01^{\mathrm{g}}$ & $10.78^{\mathrm{h}}$ & $39.79^{\mathrm{h}}$ & $2.60^{\mathrm{h}}$ & $6.35^{\mathrm{h}}$ & $4.26^{\mathrm{g}}$ & $13.21^{\mathrm{e}}$ \\
\hline FDA & $6.13^{\text {hi }}$ & $12.30^{\mathrm{ij}}$ & $6.24^{\mathrm{i}}$ & $24.67 \mathrm{i}$ & $2.50^{\mathrm{h}}$ & $3.42^{\mathrm{i}}$ & $3.06^{\mathrm{h}}$ & $8.98^{g}$ \\
\hline GTS & $5.54 j$ & $19.76^{\mathrm{h}}$ & $7.49^{\mathrm{i}}$ & $32.79^{\mathrm{i}}$ & $1.80^{\mathrm{i}}$ & $7.42^{\mathrm{g}}$ & $2.57^{\mathrm{i}}$ & $11.19^{\mathrm{ef}}$ \\
\hline Green life & $16.35^{\mathrm{f}}$ & $28.45^{\mathrm{e}}$ & $23.98^{\mathrm{g}}$ & $68.78^{\mathrm{f}}$ & $3.19 \mathrm{~g}$ & $10.73^{\mathrm{f}}$ & $7.33^{f}$ & $21.25^{\mathrm{d}}$ \\
\hline Kaji & $25.66^{\mathrm{d}}$ & $26.95^{\mathrm{f}}$ & $27.59^{\mathrm{f}}$ & $80.20^{\mathrm{e}}$ & $5.06^{\mathrm{e}}$ & $17.89^{c}$ & $7.78^{\mathrm{e}}$ & $30.73^{c}$ \\
\hline Majim & $28.59^{b}$ & $51.78^{\mathrm{b}}$ & $40.50^{\mathrm{b}}$ & $120.87^{c}$ & $14.02^{c}$ & $20.58^{\mathrm{b}}$ & $15.4^{\mathrm{a}}$ & $50^{\mathrm{b}}$ \\
\hline Shebok & $23.95^{\mathrm{e}}$ & $43.39^{d}$ & $37.99^{c}$ & $105.34^{\mathrm{d}}$ & $10.46^{\mathrm{d}}$ & $12.63^{\mathrm{d}}$ & $8.86^{\mathrm{d}}$ & $31.95^{c}$ \\
\hline Shufola & $6.25^{\mathrm{h}}$ & $12.14^{j}$ & $33.75^{\mathrm{e}}$ & $52.14^{\mathrm{g}}$ & $3.76^{\mathrm{f}}$ & $2.88^{\mathrm{i}}$ & $7.90^{\mathrm{e}}$ & $14.54^{\mathrm{e}}$ \\
\hline Cowdung & $40.70^{\mathrm{a}}$ & $48.68^{c}$ & $36.70^{\mathrm{d}}$ & $126.08^{\mathrm{b}}$ & $15.04^{\mathrm{b}}$ & $12.30^{\mathrm{e}}$ & $11.48^{c}$ & $38.82^{\mathrm{bc}}$ \\
\hline Water hyacinth & $26.12^{c}$ & $60.05^{\mathrm{a}}$ & $43.02^{\mathrm{a}}$ & $129.19^{a}$ & $16.04^{\mathrm{a}}$ & $27.68^{\mathrm{a}}$ & 14.25 & $57.97^{a}$ \\
\hline
\end{tabular}

a b cdef $\mathrm{gh}$ Ij Data bearing different superscripts within the same column differ significantly at $5 \%$ level.

The study inferred that growth attributes of Malabar nut increased in water hyacinth compost, which saved natural resources soil and promoting effectively modern efficient ecological agriculture.

\section{References}

1. Kumar AJ, RM Ram and SM Kumar 2005. Modulatory influence of Adhatoda vasica Nees leaf extract against gamma irradiation in Swiss albino mice. Phytomedicine 12: 285-293.

2. Joshi BS,Y Bai and MS Puar 1994. ${ }^{1} \mathrm{H}$ and ${ }^{13} \mathrm{C}$ NMR assignments for some pyrroloquinoline alkaloids of Adhatoda vasica. J. Natural Product 57: 553-662.

3. Chopra RN 1982. Indigenous Drugs of India. Academic Publishers, Kolkata, India. pp. 816.

5. Ahmad S, M Garg, M Ali, M Singh, MT Athar and SH Ansari 2009. A phyto-pharmacological overview on Adhatoda zeylanica. Medic. Syn. Adhatoda vasica (Linn.) Nees. Nat. Prod. Rad. 8: 549-554. 
6. Bhat VS, DD Nasavat and BR Mardikar 1978. Adhatoda vasica-an ayurvedic plant. Indian Drugs 15: 62-66.

7. Dymock W 1972. India pharmacographia of plants. Hamdard National Foundation of Pakistan 3: 343-344.

8. Atal CK 1980. Chemistry and pharmacology of vasicine: a new oxytocin and abortifacient. Indian Drugs 15: 15-18.

9. Adnan M, J Hussain, MT Shah, F Ullah, JK Shinwari, A Bahadar and AL Khan 2010. Proximate and nutrient composition of medicinal plants of humid and sub-humid regions in Northwest Pakistan. J. Med. Plant Res. 4: 339-345.

10. Dymock W, CJH Waeden and D Hooper 1890. Pharmacographia Indica: A History of the Principal Drugs of Vegetable Origin Met within British India. Paul, Trech, Trubner and Co. Ltd., London. p. 50-54.

11. Heikamp F, J Raupp and B Ludwig 2011. Soil organic matter pools and crop yields as affected by the rate of farmyard manure and use of biodynamic preparations in a sandy soil. Organic Agric.11:111-124.

12. Premsekhar M and V Rajashree 2009. Influence of organic manures on growth, yield and quality of Okra. Am-Eurasian J. Sustainable Agric. 3(1): 6-8.

13. Walkley A and IA Black 1934. An examination of the Degtjareff method for determining soil organic matter and a proposed modification of the chromic acid titration method. Soil Science 37: 29-38.

14. Marr IL and MS Cresser 1983. The lithosphere. In: Environmental Chemical Analysis. Blackie and Son, UK. p. 155-182.

15. Murphy J and JP Riley 1962. A modified single solution method for the determination of phosphate in natural waters. Anal. Chim. Acta 27: 31-36.

16. Anderson JM and JSI Ingram 1989. Soil physical analysis: Field capacity. In: Tropical Soil Biology and Fertility: A Handbook of Methods. CAB International, Wallingford, Oxon OX10 8DE, UK. p. 50-51.

17. Obeid M. 1984. Water hyacinth (Eichhornia crassipes Mart.) Solms. Proceedings of the International Conference on Water Hyacinth. Hyderabad, India. p. 145-148.

18. Constantinides $\mathrm{M}$ and JH Fownes 1994. Nitrogen mineralization from leaves and litter of tropical plants- relationship to nitrogen, lignin and soluble polyphenol concentrations. Soil Biol. Biochem. 26: 49 -55. 